\title{
Process Personalization Framework for Service-driven Enterprises
}

\author{
Rajani S. Sadasivam ${ }^{1,2}$, Gayathri Sundar ${ }^{1,2}$, Murat M. Tanik ${ }^{1}$, and Murat N. Tanju ${ }^{3}$ \\ ${ }^{I}$ Department of Electrical and Computer Engineering \\ ${ }^{2}$ Division of Continuing Medical Education \\ ${ }^{3}$ Department of Accounting and Information Systems \\ University of Alabama at Birmingham \\ rajani@uab.edu
}

\begin{abstract}
Service functions and service activities are integral part of enterprises. Although technologies have improved for developing service functions, errors persist in service activities. Noted computer scientist Ramamoorthy describes personalization, customization, and humanization of service functions as an effective approach for reducing error in service activities. This paper argues that current personalization approaches does not effectively address the entire spectrum of service functions. The proposed personalization framework can advance current state of personalization through enabling tools as services and services as tools. We discuss the framework utilizing biological research as a service-driven enterprise example. The proposed framework is based on our Enterprise Process Personalization patent.
\end{abstract}

Keywords: Services, Personalization, Processes, Cyberinfrastructures

\section{Service-driven enterprises}

"An American can have a Ford in any color so long as it's black."

- Henry Ford on the first production Model T Ford built on September 27, 1908

Ubiquitous Internet has created fundamental changes in the operation of enterprises. The principle challenge is to keep up with the Internet's hyper-competitive environment while also maintaining and leveraging the value of existing business systems. Thus, choosing the right software development strategy has major implications for enterprises' long-term competitive abilities [1]. Management gurus in the 50's were preaching management dimensions of planning, organization, integration, and measurement. The advancement in practical and efficient mainframe computers in the 60's allowed enterprises to implement software systems on mainframe architectures. Computer languages, databases, and all kinds of software tools became practical, powerful, and popular in the 70's. Vision, motivation, and collaboration were added to the arsenal of management techniques in the $80 \mathrm{~s}$. The 80 's decade also included transition from mainframe architecture to networked architecture. In the 90's it became apparent that all kinds of tools and systems developed in previous decades were essentially for processing data. Therefore, in the decade of 2000 development of knowledge and information management tools has taken precedence. However, these knowledge tools are only designed to improve computational efficiency. They are not effectively designed to aid knowledge workers. Ironically, at the same time, our modern enterprises are moving towards a service economy and the use of knowledge workers are on the rise.

The transformations experienced in the automotive industry exemplify the complex needs modern servicedriven enterprise. When Henry Ford rolled out his first automobile at the beginning of the $20^{\text {th }}$ century, he created an example of a modern enterprise, which offers services with little or no personalization. Ford's venture was a selfcontained exercise in efficient mass-production by implementation of the assembly line for manufacturing. However, automobiles of today have diversified in so many ways because of the forces of customization and technology advances. Automobiles have become complicated hybrids of mechanical, electrical, electronic, chemical, and software components now available in all shades of color, make, and model.

Section 2 in this paper introduces the concept of services and service functions emphasizing the layered hierarchical model. Section 3 reviews current personalization schemes found in the literature. Section 4 discusses the limitations of current personalization schemes and describes the proposed personalization framework utilizing biological research as a service-driven enterprise example.

\section{Service functions and personalization}

A service is the work performed directly or indirectly to satisfy the needs of customers [2]. These interactions with customers occur in many business contexts such as Business-to-Business, Business-to-Customer, Researcherto-Researcher, Department-to-Department, and Team-toTeam. Therefore, service functions are becoming integral part of enterprises. Ramamoorthy [2] classifies service 
functions based on six distinguishing features - Human (customer) needs driven, Knowledge-technology intensive, Automation intensive, Human interaction intensive, Information technology intensive, and Team based.

Web services and other Internet technologies have evolved allowing enterprises build service functions efficiently. Web service standards include Web services Description Language (WSDL) for describing services, Simple Object Access Protocol (SOAP) as a messaging framework, and Business-Process Execution Language (BPEL) for composing services. Web service standards also include Universal Description, Discovery, and Integration (UDDI) for publishing, finding and using Web services over the Internet. Currently, there are over sixty Web service standards [3].

Web services provide higher abstractions and enable "componentizing" service functions. Hence, enterprises are able to effectively automate their business processes producing results in an unprecedented speed [4]. However, service activities are still prone to intentional and unintentional errors because of the intensive interactions between people and machines. Ramamoorthy describes the layered hierarchical model (Table 1) as an effective approach for reducing errors in service functions. He recommends that by enhancing users' convenience, enterprises can reduce the incidence of errors, and thereby improve their productivity and quality of work. Personalization enhancements are not comprehensive in service software and enterprises must improve them for reducing service errors.

Table 1. Layered Hierarchical Model adapted from [2]

\begin{tabular}{|l|l|}
\hline Hierarchy & Characteristics \\
\hline Personalization & $\begin{array}{l}\text { Fit the users' individual preferences or } \\
\text { disabilities }\end{array}$ \\
\hline Humanization & $\begin{array}{l}\text { Enable responses of machines to be natural } \\
\text { and human-like }\end{array}$ \\
\hline Customization & $\begin{array}{l}\text { Customize or precondition general-purpose } \\
\text { machines to perform specialized } \\
\text { application based tasks efficiently and } \\
\text { effectively }\end{array}$ \\
\hline
\end{tabular}

\section{Current approaches to personalization in service-driven enterprises}

Personalization today is being used in a variety of service-driven enterprises including education and training, research, digital libraries, e-commerce site, and mobile applications. In the literature, we found personalization described in several ways with overlaps. We summarize some of them here. Reference [5] describes personalization in three ways:
- Customization: Customization is the easiest type of personalization. Customization allows users to change the "look and feel" of the user interface. It requires little intelligence on the part of the system. Example of customization can be seen in website such as MyYahoo, and CbsSportsline. Many portals come with the inbuilt ability to allow users to customize them.

- Individualization: Individualization is targeted towards individual users. The content of website is altered based on the users past behavior. Amazon.com personalized recommendations that are based on the users previous purchase history is an example of individualization.

- Group characterization: Group characterization is achieved by classifying users into groups based on certain features and then altering content based on that classification. Amazon.com also utilizes Group characterization to recommend books.

Eirinaki and Michalis Vazirgiannis [6] define process of customizing the content and structure of a Web site to the specific and individual needs of each user taking advantage of the user's navigational behavior. They outline the following process for personalization: 1) collection of Web data, (2) modeling and categorization of these data (preprocessing phase), (3) analysis of collected data, and (4) the determination of the actions that should be performed. They describe four ways of implementing personalization:

- Rule-based filtering: Users are asked a set of questions tailoring to the user needs based on his answers.

- Content-based filtering: Content-based filtering is similar to individualization.

- Collaborative filtering: Collaborative filtering is similar to group characterizations.

- Web usage mining: It is the application of statistical and data mining methods to the Web log data resulting in a set of useful patterns that indicate users' navigational behavior.

Rossi et. al. [7] classify personalization adopting an Object-Oriented Hypermedia Design Method designcentered view on the structure and contents of the nodes and link topology. The classify personalization in the following ways:

- Static Customization: The customization is fully defined at design time. It requires either the customer identifies himself or different entry points be given to different users.

- Link and Content Personalization: Link personalization is described as selecting the links that are more relevant to the user, thereby, changing the original navigation space by reducing or improving the relationships between nodes. Content 
personalization is described as nodes (pages) presenting different information to different users.

- Structure Customization: Structure Customization allows users to see from a repertoire of options. Most of the options also allow customizing the interface layout as well. MyYahoo is cited as an example allowing users to choose first which modules the user will get in his site (e.g., Weather, Headlines, Financial, etc) and then which information he wants to see within each module

- Context Personalization: Personalizing navigational contexts is critical when the same information (node) can be reached in different situations. The cited example is the Conference Paper Review Application in which papers can be accessed in different contexts either the whole set of papers, papers that were reviewed by a person, papers in a particular topic, or accepted papers.

- Reusing Specifications: In many software applications, different user profiles may share part of the same specifications and share the same information across the system. The cited example is the online $\mathrm{CD}$ store example, in which both customers' and managers' users specifications exhibit similar information with just small variations.

Wu. et al. [8] incorporates Rossi's recommendation into a higher level framework of software approaches based on a user perspective adding few more types of personalization. According to their scheme, there are two main categories of personalization namely the user-driven (implicit) and explicit (Table 2).

Table 2. Wu et. al. personalization framework [8]

\begin{tabular}{|c|c|c|}
\hline Implicit & $\begin{array}{l}\text { Interface configured by } \\
\text { computer. } \\
\text { Examples: Cookies that } \\
\text { provide a personal } \\
\text { welcome with user's } \\
\text { name; Opportunistic } \\
\text { links that generate } \\
\text { additional } \\
\text { advertisements for a } \\
\text { travel destination }\end{array}$ & $\begin{array}{l}\text { Content configured } \\
\text { by computer. } \\
\text { Example: } \\
\text { Collaborative } \\
\text { filtering } \\
\text { recommendations for } \\
\text { book purchases based } \\
\text { on prior buyers' } \\
\text { purchases }\end{array}$ \\
\hline Explicit & $\begin{array}{l}\text { Interface configured by } \\
\text { users. } \\
\text { Examples: Profile- } \\
\text { based personalization } \\
\text { that removes graphics } \\
\text { from displays to save } \\
\text { user download time, } \\
\text { personal tools such as a } \\
\text { personal calendar }\end{array}$ & $\begin{array}{l}\text { User-configured } \\
\text { content } \\
\text { customization. } \\
\text { Example: Content } \\
\text { filtering } \\
\text { recommendations for } \\
\text { a video based on a } \\
\text { user-provided profile }\end{array}$ \\
\hline
\end{tabular}

Wu. et al. also [8] introduce Anthropomorphic Personalization. Anthropomorphic Personalization is described as customizing information systems to act like a human. Anthropomorphic Personalization is similar to humanization in Ramamoorthy's layered hierarchical model. Mulvane et. al. [10] summarized four useful articles tabularized by $\mathrm{Wu}$ [9] (Table 3) that shows different data mining ideas for personalization systems.

\section{Table 3. Mulvenna et. al. personalization ideas summary articles $[9,10]$}

\begin{tabular}{|c|c|}
\hline $\begin{array}{l}\text { Article } \\
\text { Authors }\end{array}$ & $\begin{array}{l}\text { Ideas to data mining in terms of } \\
\text { personalization }\end{array}$ \\
\hline Spiliopoulou & $\begin{array}{l}\text { 1. A rationale for why Web log data should be } \\
\text { mined. } \\
\text { 2. The effectiveness of a Web site in providing } \\
\text { users with the content they need in the most } \\
\text { optimized manner is the key to retaining them. } \\
\text { 3. A process by which mining for navigational } \\
\text { patterns may be used to gain insight into a Web } \\
\text { site's usage and optimality with respect to its } \\
\text { user population. }\end{array}$ \\
\hline $\begin{array}{l}\text { Cingil, Dogac, } \\
\text { and Azgin }\end{array}$ & $\begin{array}{l}\text { 1. The need for interoperability when mining the } \\
\text { Web } \\
\text { 2. How the various W3C standards can be used } \\
\text { to achieve personalization applications. } \\
\text { 3. How the recent data exchange, metadata and } \\
\text { privacy standards from the World Wide Web } \\
\text { Consortium (W3C), namely, Extensible Markup } \\
\text { Language (XML), Resource Description } \\
\text { Framework (RDF), and Platform for Privacy } \\
\text { Preferences (P3P) may be used to support } \\
\text { personalization activities. }\end{array}$ \\
\hline $\begin{array}{l}\text { Mobasher, } \\
\text { Cooley, and } \\
\text { Srivastava }\end{array}$ & $\begin{array}{l}\text { 1. A framework for mining Web log files to } \\
\text { discover knowledge for the provision of } \\
\text { recommendations to current users based on their } \\
\text { browsing similarities with previous users. } \\
\text { 2. The process for discovering such knowledge } \\
\text { includes gathering and preprocessing the data } \\
\text { necessary for discovering user behaviors, } \\
\text { application of data mining techniques to } \\
\text { discover usage patterns, and aggregation and } \\
\text { filtering of the data mining results in order to } \\
\text { create decision rules for customizing Web site } \\
\text { content based on an individual user's behavior. }\end{array}$ \\
\hline $\begin{array}{l}\text { Perkowitz, } \\
\text { Etzioni }\end{array}$ & $\begin{array}{l}\text { 1. Personalization as a process that adapts an } \\
\text { Internet site through the automated generation of } \\
\text { index pages for the Web site. } \\
\text { 2. Adaptive Internet sites were explored in this } \\
\text { paper. Those sites automatically improve their } \\
\text { organization and presentation by learning from } \\
\text { visitor access patterns. Adaptive Web sites mine } \\
\text { the data buried in Web server logs to produce } \\
\text { more easily navigable Web sites. }\end{array}$ \\
\hline
\end{tabular}




\section{Proposed Approach: Integration of Personalization into the Workplace}

Although we find personalization widely used, the impact of personalization is still debated. For example, Kobsa [11] predicts that personalization is going to a play a big role in future websites. However, the Jupiter research report "Beyond the Personalization Myth" [12] describes personalization as expensive and unproductive. They argue that companies must rethink personalization, and pursue lower-cost alternatives. Many in the industry argue that personalization is inefficient because it is very difficult to predict human behavior and automate it. Additionally, considering the list of service functions [2] we find that the current state of personalization schemes minimally address them. Most of the personalization is targeted only towards web pages and some Business-to-Customer interactions and generally ignores other types of service interactions. However, as Ramamoorthy describes, we will continue to be error-prone in service activities until we rethink our design strategies to effectively integrate personalization into the workplace [2].

Recently, few papers appeared discussing personalized selection of services [13,14]. Semantic tools [15] are being developed to aid the discovery of services based on user's preferences. This, we argue, is an important step towards integrating personalization into the workplace (that is into service functions) and therefore these techniques should be viewed in that context. If we can personalize the use of tools allowing users to utilize different tools and reuse tool functions across different service activities, we can make significant progress towards reducing errors in them. Our proposed framework can integrate personalization into the workplace through two mechanisms namely tools as services and services as tools. The above two mechanisms allow users to continue using their desired tools and functions and thus enhance their service activities. The proposed personalization framework is based on our Enterprise Process Personalization (EPP) patent [16].

\subsection{Biological research example as a service- driven enterprise: A personalization viewpoint}

Biological research serves as a good example of a service-driven enterprise for our discussion. Biological research has been greatly enhanced as a service-driven enterprise by the Human Genome Project [17]. The availability of genome sequences for hundreds of different biological species has created a foundation for development of a broad array of high-throughput experimentation tools and methods for automated generation of biological data. Understanding the emergent properties of "gene networks" such as regulated gene expression, and organization of genes, proteins, enzymes, and metabolites into pathways is a major challenge, spanning many different scientific disciplines [18]. Although High-Dimensional Biology is a relatively new area, a staggering accumulation of data has resulted in integration being a bottleneck for understanding [17]. Consequently, a great number of service cyberinfrastructures such as MyGrid, Biomedical Informatics Research Network (BIRN), and BioMoby have also been developed [19] . However, the adoption of these service cyberinfrastructures by researchers of biology and medicine is almost negligible. Buetow [19] suggests that the reason is biology end-user really doesn't care what technologies underlie cyberinfrastructure. However, the biomedical end-user does provide key requirements that should be taken into consideration when choosing technology. He suggests, facilitating adoption, cyberinfrastructure should be an extension of or interoperate with infrastructure already available to users. Many biologists currently use "rich" end-user tools to carry out their data analysis. They use tools such as Microsoft Excel for formatting data, Matlab, or other in house software for analysis. Unfortunately, the cyberinfratructures replace many of these functions and interactive tasks with web pages. Web pages provide excellent virtual presence but they require constant maintenance in the form of writing client scripts to present a rich and interactive environment. As Buetow suggests, biologist would like to continue either using the same tools or as a minimum a tool, which provides a rich user environment as their current tools. They are interested in the possibilities offered by the cyberinfratructures but the new web pages and the associated learning curve acts as a barrier to adoption. Although our experience has been in interfacing with biological research groups, the above user scenario holds true across many service-driven enterprises.

\subsection{Personalization framework for service-driven enterprises}

Our EPP patent [16] identifies service-enabled-tools and tools as services as two important personalization mechanisms. The service-enabled-tools approach would allow users to choose the tools they prefer and utilize the cyberinfrastructures to enhance their current work. For example, biological researchers who prefer Excel as their tool would be able to generate data in Excel format, invoke the cyberinfrastructures to augment their research, and then generate output curves or plots in Excel. The users would not have to move from Excel to another application to invoke the cyberinfrastructures and then come back to Excel or another application to generate the curves. The advantage of this approach is that many tools already have Web service capability, which can be used to enable them. In addition, the users are familiar with the environment and the learning curve would be minimal or nonexistent. The disadvantage of this approach is if the number of tools is very high, then it becomes difficult to configure and service-enable them individually. In the tools-as-services 
approach the different tools and their functions would be componentized using service descriptions and placed in a repository. Tools would then query the repository as they would query a repository like UDDI and invoke the tool services. Interestingly, the tools-as-services approach could be used to enhance the service-enabled-tools approach. In the service-enabled-tools biology example, the user could invoke Matlab function to perform some data processing and then generate the output that could be displayed in Excel. The user still utilizes the familiar Excel environment, but he has greatly enhanced his efficiency by using the more powerful and also familiar Matlab functions. Alternatively, if the enterprise does not want to create service-enable-tools, rich-user clients can be developed using technologies such as Smart Clients, AJAX, and Macromedia Flex which can all communicate through Web services. Another way to use the proposed architecture is to develop plugins to incorporate the feature into familiar environments. This approach is not new. For example, many Java tools such as Oracle BPEL designer are being developed as plugins to Eclipse which is a popular Java IDE. The difficulty with this architecture is tools are not by default componentized using service descriptions. Therefore, enterprises have to undertake efforts to convert them as services. However, if this model becomes popular than it is natural to expect future vendor companies will enable their tools as services. Evidence can be seen in current portlets approach such as JSR-portlets (http://www.jcp.org/aboutJava/communityprocess/review/js r168/) and WSRP (http://www.oasisopen.org/committees/tc_home.php?wg_abbrev=wsrp) that allow componentizing Web applications even their user interface. Similarly, we can expect tools specifications, which allow their reuse across different applications.

The proposed personalization framework based on the Enterprise Service Bus (ESB) and Service Oriented Architecture (SOA) pattern is depicted in Figure 1. ESB is described as a highly distributed approach to enterprise integration approach that provides capabilities for building integrated systems in incremental, digestible chunks, maintaining their own local control and autonomy, while still being able to connect together each integration project into a larger, more global integration fabric, or grid [20]. We have incorporated ESB to allow enterprises to connect to the dissimilar cyberinfrastructures. For example, in the biology domain there are WSDL services, OWL-S services, MyGrid services, BioMoby services. In the registry layer, we also have included tool-service registries to publish tools that are componentized as Services. We are just differentiating the tool-service registries from the service registries conceptually but in actual implementation, they could be the same. We have extended the ESB conceptually with a personalization mediator service [15]. The mediator service mediates the mismatches the tool functions to enable their use as services. The mismatches could be caused by the different architectural, design, and implementation decisions within each component create the mismatch that leads to the lack of integration. Also in the framework are the Business Process Management layer and Business Process Orchestration layer [21]. Business process orchestration supports the composition of multiple services, tools, other objects into more complex processes. BPEL, WSCI or other technologies can be used for composition. Business process management allows the enterprise to manage the composed processes.

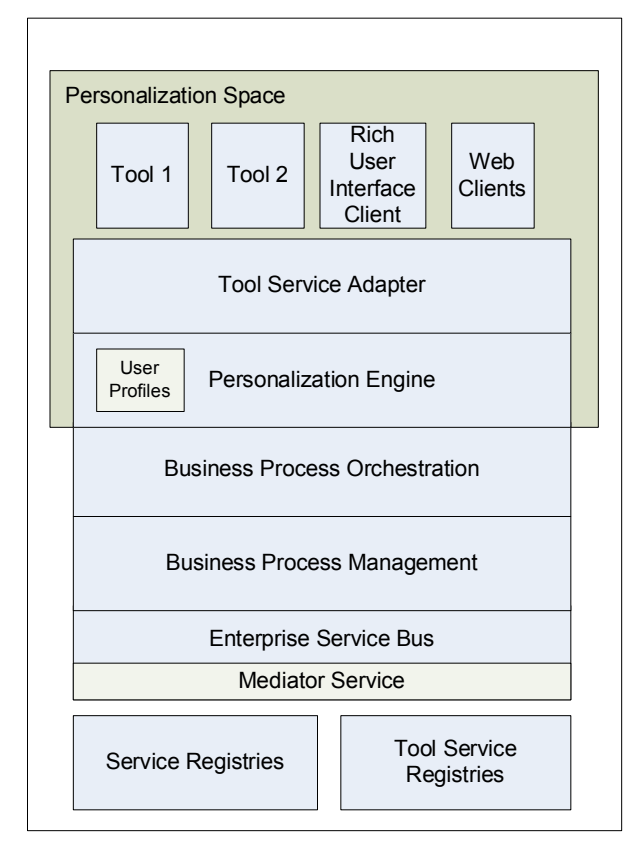

\section{Figure 1. Proposed Process Personalization Framework}

Enterprises could add personalization approaches to provide recommendations, context personalization, collaborative personalization, or others personalization found in the literature. Users profile is also included in the personalization engine. Semantic-based approaches such as OWL (http://www.w3.org/TR/owl-features/) or FOAF (http://xmlns.com/foaf/0.1/) can be used to enhance the description of profiles. Users should also be able to authenticate into one tool and be able to navigate to the others seamlessly. Therefore, another issue to address in the above architecture is single-sign-on feature. Many singlesign-on approaches have been proposed which could be incorporated into the system [22]. Additionally, as mentioned before many tools are now offering Web Service integration. We can also integrate with tools that do not offer Web Service extensions by writing tool-service adapters. Support for other Web Service standards like WStransaction, WS-security, WS-policy, WS-reliable messaging have not be shown in the framework for simplicity but they will be included as needed [21]. 


\section{Conclusion and future work}

The proposed framework in this paper is based on our EPP patent [16] that identifies two personalization mechanisms. The two mechanisms are service-enabledtools and tools-as-services. Currently, work is underway developing a personalization service infrastructure for a genetics research laboratory. We are developing toolservice adapters to develop service-enabled-tools needed for particular genetics research. We are also working on semantic mediator approaches to "componentize" some of the genetics research tools.

\section{Acknowledgment}

We acknowledge the immense contributions of Dr. Ramamoorthy to our Service-driven enterprise research. We are especially grateful for his continuing support and personal attention to our research. The paper is part of Rajani's dissertation effort and Dr. Ramamoorthy is a member of his dissertation committee. We thank Dr. Hartman of the Department of Genetics for collaborating with us to develop his biological research infrastructure. We thank Dr. Jololian of the Computer Science Department for collaborating on our component integration research. We also thank the Division of Continuing Medical Education for their support and contribution.

\section{References:}

[1] M. M. Tanik, P. NG, and R. T. Yeh, "Electronic Enterprise Engineering - A 21st century discipline for a new generation of engineers," NJIT Research, pp. 6-11, 1997.

[2] C. V. Ramamoorthy, "A Study of the Service industry Functions, Features, and Control," ICICE Transactions Communications, vol. E83-B, pp. 885-903, 2000.

[3] 2005, Industry Standards Overload: Making Sense of WSDL, SOAP, WS-*, and more!, 2005, 12/1/05, http://beaworldonline.bea.com/files/subsystems/12077/BEA-65697.pdf, D. Orchard and J. Joergensen

[4] A. H. Dogru and M. M. Tanik, " Process Model for Component-Oriented Software Engineering," IEEE Software, vol. 0740-7459/03/, pp. 2-9, 2003.

[5] 2006, Personalization, 2006, January, http://www.medill.northwestern.edu/imc/studentwork/pr ojects/Sticky/personalization.htm,

[6] M. Eirinaki and M. Vazirgiannis, "Web mining for web personalization http://doi.acm.org/10.1145/643477.643478 " ACM Trans. Inter. Tech. , vol. 3 pp. 1-27 2003

[7] G. Rossi, D. Schwabe, and R. Guimaraes, "Designing personalized web applications http://doi.acm.org/10.1145/371920.372069," in

Proceedings of the 10th international conference on
World Wide Web. Hong Kong, Hong Kong: ACM Press, 2001, pp. 275-284.

[8] D. Wu, I. Im, M. Tremaine, K. Instone, and M. Turoff, "A framework for classifying personalization scheme used on e-commerce Websites," in proceedings of $36 t h$ Annual Hawaii International Conference on System Sciences, 2003

[9] 2001, Personalization in user interface, 2006, January, http://eies.njit.edu/ turoff/coursenotes/CIS732/samplepr o/CIS732FinalProject Dezhi.htm, D. Wu and M. Turoff

[10] M. D. Mulvenna, S. S. Anand, and A. G. Büchner, "Personalization on the Net using Web mining: introduction http://doi.acm.org/10.1145/345124.345165," Commun. $A C M$, vol. 43, pp. 122-125, 2000.

[11] A. Kobsa, "Tailoring Privacy to Users' Needs " in Proceedings of the 8th International Conference on User Modeling 2001 Springer-Verlag, 2001 pp. 303-313

[12] M. Berk, J. Blank, D. Daniels, and D. Schatsky, Beyond the Personalization Myth: Cost-effective Alternatives to Influence Intent: JupiterResearch, 2003.

[13] W.-T. Balke and M. Wagner, "Towards Personalized Selection of Web Services," in proceedings of $W W W$ 2003, Budapest, Hungary, 2003, May 20-24.

[14] M. Paolucci and K. Sycara, "Autonomous Semantic Web Services," IEEE Internet Computing, vol. 7, pp. 34- 41, 2003.

[15] L. K. Jololian, "Towards Semantic Integration of Components using a Service-Based Architecture," in proceedings of IDPT 2005, Beijing, China, 2003, June $12-16$.

[16] R. Sadasivam, M. Tanik, and L. Jololian, "US Provisional Patent Application: Drag-and-Drop Communication of Data via a Computer Network," 2005.

[17] T. V. Venkatesh and H. B. Harlow, "Integromics: challenges in data integration," Genome Biol, vol. 3, pp. REPORTS4027, 2002.

[18] M. E. Csete and J. C. Doyle, "Reverse engineering of biological complexity," Science, vol. 295, pp. 1664-9, 2002.

[19] K. H. Buetow, "Cyberinfrastructure: empowering a "third way" in biomedical research," Science, vol. 308, pp. 821-4, 2005.

[20] D. A. Chappell, Enterprise service bus, 1st ed. Sebastopol, Calif:: O'Reilly, 2004.

[21] M. B. Juric, B. Mathew, and P. Sarang, "Business Process Execution Language for Web Services: BPEL and BPEL4WS," Packt Publishing, 2004, pp. 270.

[22] R. S. Sadasivam, M. M. Tanik, J. Gemmill, and T. Jannett, "Cyberinfrastructure Development - A Component Based Approach with Software Agents," in proceedings of IDPT 2003, Austin, Texas, 2003, December 3-5. 